\title{
An Unusual Transformation of a Nonseminomatous Germ Cell Tumor to Adenocarcinoma
}

\author{
Jennifer N. Leibovitch ${ }^{\mathrm{a}, \mathrm{d}}$, Poornima Ramadas ${ }^{\mathrm{b}}$, Yelena Fudym ${ }^{\mathrm{c}}$, Sam Benjamin ${ }^{\mathrm{b}}$
}

\begin{abstract}
Transformation of germ cell tumor to an alternate malignancy is rare; and it is believed to be secondary to teratomatous elements of the initial tumor surviving chemotherapy and subsequently proliferating. Here, we describe a patient with transformation of a nonseminomatous germ cell tumor to adenocarcinoma. A 55-year-old male with history of nonseminomatous germ cell tumor of the left testicle, previously resected, followed by four cycles of BEP chemotherapy, on active surveillance, presented nearly 20 years later with nausea and rising alpha-fetoprotein levels. Computed tomography (CT) abdomen revealed bulky retrocrural lymphadenopathy with increased fluorodeoxyglucose (FDG) uptake on positron emission tomography (PET) scan. Biopsy revealed metastatic adenocarcinoma, staining strongly positive for SALL4 and CDX2, with focal rare positivity for CK20 in the tumor cells, consistent with germ cell origin. FOLFOX therapy was therefore initiated. This is one of few documented cases showing transformation of nonseminomatous germ cell tumor to adenocarcinoma, for which surgical resection is favored as primary therapy. However, aggressive adjuvant chemotherapy may be considered and should be directed towards the most aggressive found histology.
\end{abstract}

Keywords: Nonseminomatous germ cell tumor; Transformation; Metastatic adenocarcinoma; SALL4; CDX2; CK20; Germ cell; Isochromosome $12 p$

\section{Introduction}

Testicular germ cell tumor (GCT) comprises around $1 \%$ of all cancers in men. However, it is the most common solid malignancy that affects young males between 15 and 35 years. GCT

Manuscript submitted April 18, 2019, accepted April 29, 2019

${ }^{a}$ Department of Medicine, Upstate Medical University, Syracuse, NY, USA bDivision of Hematology/Oncology, Department of Medicine, Upstate Medical University, Syracuse, NY, USA

'Department of Pathology, Upstate Medical University, Syracuse, NY, USA

${ }^{\mathrm{d}}$ Corresponding Author: Jennifer N. Leibovitch, Department of Medicine, Upstate Medical University, Room 5142, 750 East Adams Street, Syracuse, NY 13210, USA.Email: leibovij@upstate.edu

doi: https://doi.org/10.14740/jmc3301 can be broadly divided into seminoma or nonseminomatous GCT (NSGCT) which includes embryonal carcinoma, choriocarcinoma, teratoma, yolk sac tumor and mixed GCT. It is one of the most curable solid malignancies with a 5-year survival rate of more than 95\% reported in 2017.

Transformation of GCT to an alternate malignancy is rare and occurs in around $2.7-8.6 \%$ of all germ cell tumors [1]. This transformation is posited to be secondary to teratomatous elements of the initial tumor surviving chemotherapy and subsequently proliferating to a different histology, although definitive evidence of this process is yet to be elucidated. This is more common with primary mediastinal non-GCTs as compared to gonadal germ cell tumors [2] Transformation to sarcoma is most common, followed by adenocarcinoma and primitive neuroectodermal tumor [3].

Here, we describe a patient with the infrequently reported transformation of a NSGCT to adenocarcinoma.

\section{Case Report}

Our patient is a 55-year-old male with a past medical history of NSGCT of the left testicle which was diagnosed in late 1981. He had elevated alpha-fetoprotein (AFP) at that time and underwent left-sided orchiectomy and retroperitoneal lymph node dissection, after which the AFP normalized. Surveillance imaging in early 1982 demonstrated a $2-\mathrm{cm}$ right lower lung lesion and a 3-cm left upper lung lesion along with elevation of AFP. He was initially treated with four cycles of bleomycin, vinblastine, and cisplatin, followed by biopsy of the lung lesions, which revealed scar tissue. Subsequent imaging showed a striking decrease in size of these lesions, rendering them difficult to identify. He was continued on surveillance and was noted to have rising AFP to 21.8 in mid-2002. Imaging studies revealed a right-sided pelvic tumor, $8 \times 6 \mathrm{~cm}$ in size, just above the previous dissection site, suggestive of metastatic disease, given his history of testicular NSGCT. He underwent complete resection of the lesion and pathology revealed adenocarcinoma. Immunohistochemistry stains were negative for AFP and human chorionic gonadotropin (HCG) but positive for carcinoembryonic antigen (CEA). His AFP again normalized after surgical resection. He was continued on active surveillance, until he began experiencing intermittent nausea, with a subsequent rise in AFP to 5.7 in early 2018. Computed tomography (CT) abdomen revealed bulky retrocrural lymphadenopathy to $3.3 \mathrm{~cm}$, as well as a $1.5-\mathrm{cm}$ left lower lobe irregularly shaped pulmonary nodule. Posi- 


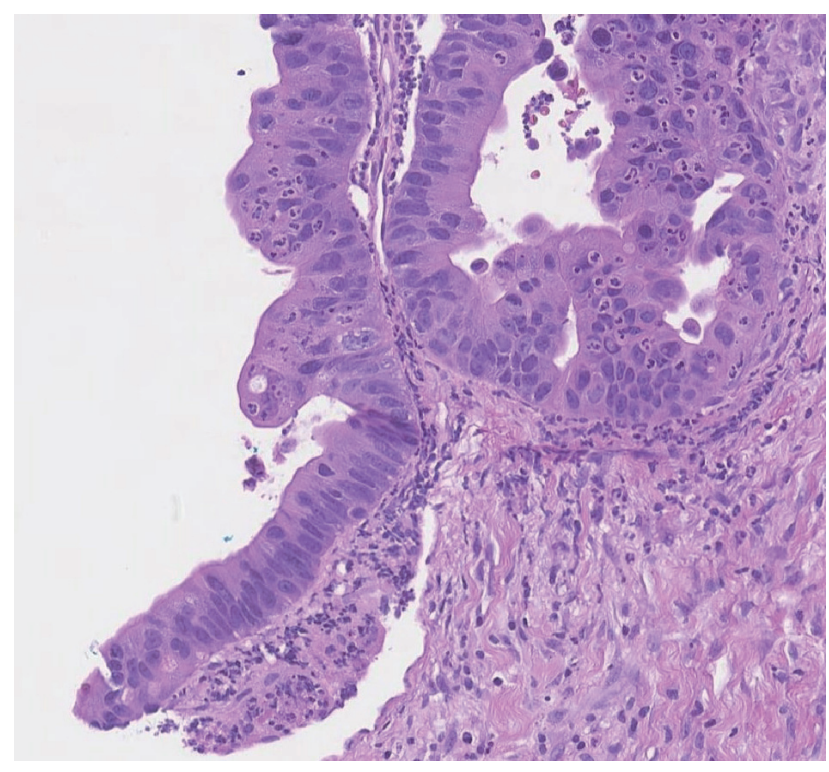

Figure 1. H\&E high power view of pathology consistent with adenocarcinoma.

tron emission tomography (PET) scan revealed aortocaval lymphadenopathy, right retrocrural and right perirenal fluorodeoxyglucose (FDG)-avid soft tissue masses with standard uptake values (SUVs) of 7.9 and 6 respectively, and an FDGavid focus in the left lower lobe measuring $1.2 \mathrm{~cm}$ with SUV of 3.3, concerning for metastasic disease versus primary lung malignancy. Given the patient's remote history of testicular GCT and metastatic adenocarcinoma in the retroperitoneal and retrocrural space combined with the recent rise in serum AFP from the patient's baseline, metastatic GCT with malignant transformation to adenocarcinoma was suspected. CTguided retrocrural mass core biopsy was then pursued and pathology was consistent with metastatic adenocarcinoma (Fig. 1), staining strongly positive for SALL4 (Fig. 2) and CDX2 (Fig. 3), with focal rare positivity for CK20 in the tumor cells. CK7, Oct3/4, and TTF-1 immunostains were negative. Chemotherapy was initiated with 5-fluorouracil, oxaliplatin,

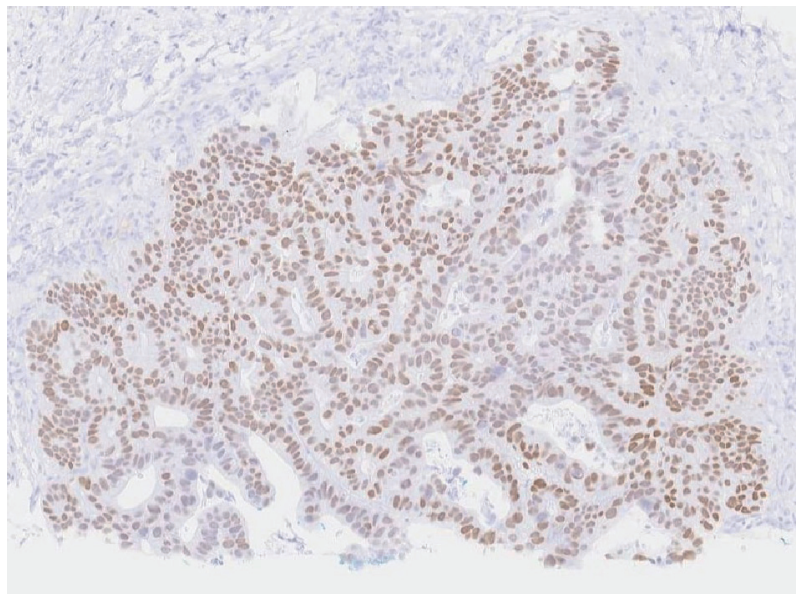

Figure 2. Tumor cells staining strongly positive for SALL4.

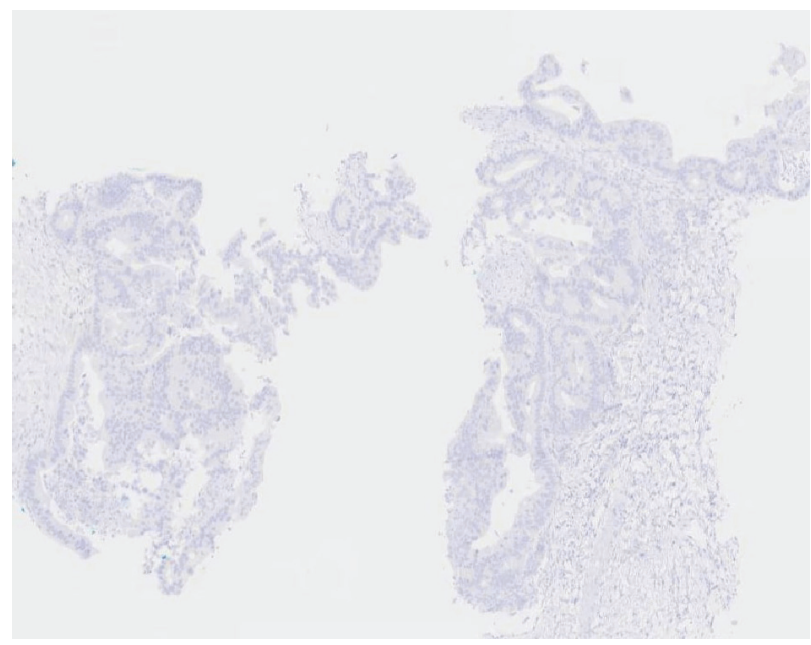

Figure 3. Tumor cells staining strongly positive for CDX2.

and leukovorin per FOLFOX regimen, with plan for surgical debulking based on response. He ultimately underwent two cycles of FOLFOX, following which a biopsy of the left lung nodule visualized on PET scan was performed. Surgical pathology demonstrated adenocarcinoma with mostly acinar growth, positive for TTF1 and negative for SALL-4 which was different in histology when compared to the retrocrural mass. This was consistent with a separate lung primary for which he underwent stereotactic body radiation treatment. He is continued on FOLFOX regimen at this time.

\section{Discussion}

Transformation to a secondary malignancy from a primary germ cell tumor is an incredibly rare phenomenon. It has been noted that most of these tumors occur in the mediastinum and retroperitoneum, and have been increasing in incidence over the past number of decades [4]. One school of thought has proposed that chemotherapy-resistant cell populations may selectively proliferate following initial chemotherapy directed at the germ cell tumor itself, as chemotherapy preferentially affects undifferentiated cells, thereby leaving differentiated mature cells behind [4]. Others have proposed that pluripotent embryonal cells within the initial carcinoma cause the malignant transformation, further supported by the presence of isochromosome $12 p$, a marker seen in a significant percentage of germ cell tumors demarcating germ cell tumor clonality in many of the transformed malignancies [3, 4]. However, the pathogenesis of this process has not been extensively researched, and is therefore currently not well understood.

The histology of these malignancies is even more difficult to delineate. A number of case studies have noted mixed germ cell patterns occurring in a large number of patients with teratoma with malignant transformation [4]. When transformed to a secondary malignancy, sarcoma was noted to be the most frequent histology, with rhabdomyosarcoma as the predominant subtype, followed by adenocarcinoma and primitive neu- 
roectodermal tumor [3]. Histologically, these tumors resemble typical primary neoplasms. However, with the use of fluorescence in situ hybridization (FISH), isochromosome $12 \mathrm{p}$ can often be identified, confirming germ cell tumor origin despite morphologic dissimilarity [5].

Surgical resection is favored as primary therapy in those presenting with germ cell tumors transformed to an alternate malignancy, especially in patients with localized disease [6]. However, radical resection is favored in those with metastatic disease as well. Adjuvant chemotherapy and radiation therapy are currently not recommended, though may be considered later in the disease course based on the pathology of the transformed component, and should be directed towards the most aggressive found histology $[3,6]$. In those with unresectable disease, prognosis is generally poor [3]. Adenocarcinomas and primitive neuroectodermal tumors are typically associated with increased resistance to both chemotherapy and radiation therapy, thus necessitating aggressive surgical intervention especially in these cases. Motzer et al demonstrated that $68 \%$ of patients in their analysis experienced a complete response to cisplatin-containing combination chemotherapy for treatment of their GCT, with an incomplete response noted in 32\%. Of the $15 \%$ of patients receiving non-platinum chemotherapy directed at the malignant transformation, none achieved complete response [3]. When initiated, chemotherapy regimens including cisplatin, for both initial and salvage treatment, are most effective, though aggressive surgical resection remains the mainstay of treatment.

Prognosis of germ cell tumors with transformation to an alternate malignancy is poor, especially in the setting of metastatic disease, with estimated median survival of about 9 months [7]. The amount of time from the initial diagnosis of germ cell tumor was noted to be a poor prognostic factor, with inferior survival noted when malignant transformation occurred more than 2 years after GCT diagnosis [4]. Better overall survival has been noted when the primary tumor originated in the gonads, as well as with fewer prior chemotherapy regimens, and transformed histology to non-primitive neuroectodermal carcinoma [4]. Complete resection of the tumor also increased overall survival [3]. However, once GCT has transformed, overall survival decreases regardless of the clinical stage at the time of diagnosis [4].

\section{Acknowledgments}

All contributing individuals qualified for authorship.

\section{Financial Disclosure}

None to declare.

\section{Conflict of Interest}

None to declare.

\section{Informed Consent}

Obtained.

\section{Author Contributions}

JL wrote the manuscript with support from PR and SB. SB supervised the project and reviewed the final manuscript, as did PR. YF aided in acquisition of data.

\section{References}

1. Rice KR, Magers MJ, Beck SD, Cary KC, Einhorn LH, Ulbright TM, Foster RS. Management of germ cell tumors with somatic type malignancy: pathological features, prognostic factors and survival outcomes. J Urol. 2014;192(5):1403-1409.

2. Shaw A, Morrell M, Weissferdt A, Hayes-Jordan A, Harrison D. Malignant transformation of testicular teratoma to PNET, adenocarcinoma, and osteosarcoma with complete remission after surgery and combination chemotherapy in a young adult male. Case Rep Oncol Med. 2018;2018:8460603.

3. Motzer RJ, Amsterdam A, Prieto V, Sheinfeld J, Murty VV, Mazumdar M, Bosl GJ, et al. Teratoma with malignant transformation: diverse malignant histologies arising in men with germ cell tumors. J Urol. 1998;159(1):133138.

4. Aliotta PJ, Castillo J, Englander LS, Nseyo UO, Huben RP. Primary mediastinal germ cell tumors. Histologic patterns of treatment failures at autopsy. Cancer. 1988;62(5):982-984.

5. Dieckmann KP, Anheuser P, Gehrckens R, Wilczak W, Sauter G, Hoflmayer D. Pure testicular seminoma relapsing late with somatic type malignancy. Case Rep Oncol Med. 2017;2017:2457023.

6. Giannatempo P, Pond GR, Sonpavde G, Albany C, Loriot Y, Sweeney CJ, Salvioni R, et al. Treatment and clinical outcomes of patients with teratoma with somatic-type malignant transformation: an international collaboration. J Urol. 2016;196(1):95-100.

7. Lin C, Du Y, Li Y, Wang H, Chang J. Superior mediastinal mature cystic teratoma with gastrointestinal adenocarcinoma transformation: Report of a case. Oncotarget. 2016;7(25):38392-38397. 